ORIGINAL ARTICLE

\title{
Thymidine phosphorylase expression in tumour cells and tumour response to capecitabine plus docetaxel chemotherapy in non-small cell lung cancer
}

\author{
J-Y Han, E K Hong, S Y Lee, S M Yoon, D H Lee, J S Lee
}

J Clin Pathol 2005;58:650-654. doi: 10.1136/icp.2004.022764

See end of article for authors' affiliations

\section{Correspondence to:} Dr J S Lee, Research Institute and Hospital, National Cancer Centre, Korea, 809 Madul-dong, Ilsan-gu, Goyang-si, Gyeonggi-do, 411-769,

Korea; jslee@ncc.re.kr

Accepted for publication 29 October 2004

\begin{abstract}
Background: Thymidine phosphorylase (TP) is the key enzyme for capecitabine activation in tumour cells. Aims: To examine whether TP expression in tumour cells and stroma is predictive of the tumour response to capecitabine plus docetaxel chemotherapy in patients with advanced non-small cell lung cancer (NSCLC). Methods: Tumour samples were available from 30 of 39 patients enrolled in a previous phase II study of capecitabine/docetaxel chemotherapy in patients with advanced NSCLC. Stromal and tumour cell TP expression was evaluated by immunohistochemistry using monoclonal antibody PD-ECGF.

Results: High tumour cell TP expression was found in 13 of 30 cases and was negatively associated with stromal TP expression $(p=0.000)$. High stromal TP expression was found in 16 of 28 cases and was strongly associated with intense macrophage infiltration $(p=0.002)$, suggesting that macrophages are the major component of TP expression in the stroma. Tumour response to capecitabine/docetaxel was significantly associated with high tumour cell TP expression $(p=0.004)$ and low stromal TP expression $(p=0.009)$. Moreover, high tumour cell TP expression was significantly associated with severe hand-foot syndrome, a toxic side effect of capecitabine $(p=0.01)$. Improved survival was seen for high tumour cell and low stromal TP expression, although results were not significant ( $p=0.6$ and 0.3 , respectively).

Conclusions: In advanced NSCLC, TP expression in tumour cells and stroma is associated with tumour response to capecitabine/docetaxel chemotherapy, and might be a useful predictor of tumour response to capecitabine based chemotherapy. A large scale prospective study is needed to confirm the prognostic significance of TP expression in NSCLC.
\end{abstract}

$\mathrm{T}$ he identification of specific tumour characteristics that could improve the ability to predict the response to treatment would be useful for optimising treatment. Although several predictive factors such as oncogenes and hormone receptors have been studied, for prodrugs, such as capecitabine, which is rationally designed to generate 5fluorouracil (5-FU) selectively in tumour cells, ${ }^{1}$ concentrations of enzymes essential for their activation would be more useful predictive factors.

"The relatively high expression of thymidine phosphorylase in non-small cell lung cancer provided a rationale for the use of capecitabine in patients with this tumour"

Capecitabine is first hydrolysed by hepatic carboxylesterase to $5^{\prime}$-deoxy-5-fluorocytidine. This intermediate is then converted to $5^{\prime}$-deoxy-5-fluorouridine by cytidine deaminase in tumour cells and the liver. The third and final step involves the conversion of $5^{\prime}$-deoxy-5-fluorouridine to 5-FU by thymidine phosphorylase (TP), which is found predominantly in tumour cells. This final step appears to be rate limiting for the efficacy of capecitabine. ${ }^{12}$

Several chemotherapeutic agents affect TP activity in tumour cells, including docetaxel, paclitaxel, mitomycin C, and cyclophosphamide. ${ }^{3}$ Preclinical studies of human breast and colon cancer xenograft models have shown that docetaxel is more active in combination with capecitabine than 5-FU or UFT (an oral 5-FU derivative agent). This in vivo synergistic activity was dependent on docetaxel induced upregulation of TP. ${ }^{4}$ In fact, docetaxel induced upregulation of TP has been confirmed in women with primary breast cancer treated with preoperative docetaxel. ${ }^{6}$ Furthermore, a

\begin{tabular}{|lll|}
\hline \multicolumn{2}{|l|}{ Table 1 Patient characteristics $(\mathrm{n}=30)$} \\
\hline Characteristic & No. of patients & $\%$ \\
\hline Age (years) & & \\
Median & 58 & \\
Range & $34-72$ & \\
Sex & 15 & 50 \\
Male & 15 & 50 \\
Female & 5 & 17 \\
Histology & 25 & 83 \\
Squamous cell & 4 & 13 \\
Adenocarcinoma & 26 & 87 \\
Stage & 16 & 53 \\
IIIB & 9 & 30 \\
IV & 5 & 17 \\
Tumour response & & \\
Partial response & & \\
Stable disease & & \\
Progressive disease & & \\
\hline
\end{tabular}

phase III study demonstrated clinically relevant synergistic antitumour activity of a capecitabine/docetaxel combination over single agent docetaxel in anthracycline pretreated patients with advanced breast cancer.

Recently, we conducted a phase II study to evaluate the efficacy and safety of the capecitabine/docetaxel combination in chemonaïve patients with advanced NSCLC. Although the antitumour activity of capecitabine has not been evaluated in NSCLC, the relatively high expression of TP in NSCLC

Abbreviations: 5-FU, 5-fluorouracil; NSCLC, non-small cell lung cancer; TP, thymidine phosphorylase 


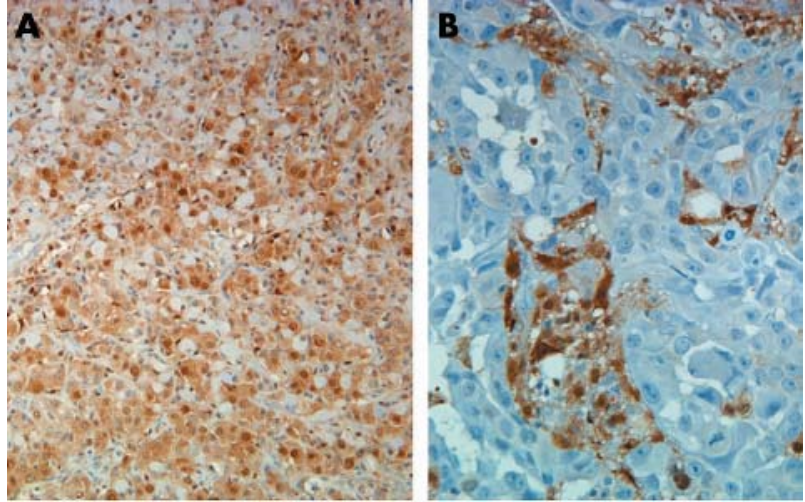

Figure 1 Representative immunohistochemical staining of thymidine phosphorylase (TP) in non-small cell lung cancer. (A) Adenocarcinoma with high TP expression in the tumour cells; there is diffuse and intense immunostaining in almost all tumour cells. (B) Adenocarcinoma with high TP expression in the stroma. Whereas stromal cells show diffuse and intense immunostaining, tumour cells are negative.

provided a rationale for the use of capecitabine in patients with this tumour. Furthermore, the confirmed clinical synergistic antitumour activity of combined capecitabine and docetaxel in advanced breast cancer ${ }^{7}$ encouraged us to conduct this trial.

Of the 39 patients enrolled, 19 partial responses were seen and the overall response rate was 53\% (95\% confidence interval, $37 \%$ to $69 \%) .{ }^{9}$ Given the fact that docetaxel alone has a single agent activity of $38 \%$ at best, and $26 \%$ on average, in chemonaïve advanced NSCLC ${ }^{10-13}$ capecitabine is thought to have significant antitumour activity against NSCLC. However, it is often associated with considerable nonhaematological toxicity, suggesting clinical synergism not only in efficacy but also in toxicity. Thus, identifying predictive factors may allow us to select patients with a high likelihood of benefiting from this combined treatment.

Because of the studies showing that TP values can affect the efficacy of capecitabine, ${ }^{3-5}$ we thought that TP expression in tumour cells and/or stroma might be a predictor of the clinical efficacy of the capecitabine plus docetaxel combination. In our present study, we investigated the immunohistochemical expression of TP in tumour cells and stroma obtained from patients enrolled in the earlier study of capecitabine plus docetaxel. We postulated that those tumours with high tumour cell TP expression would be more sensitive to capecitabine based chemotherapy than those with low TP expression.

\section{MATERIALS AND METHODS}

\section{Patients}

From December 2001 to June 2002, 39 patients with advanced NSCLC were enrolled in a phase II study of capecitabine plus docetaxel combination. We obtained formalin fixed, paraffin wax embedded bronchoscopic or needle aspiration biopsy tumour samples from 30 patients, who formed the basis of our study. Histopathological examination was performed according to the World Health Organisation classification of lung cancer. There were 25 adenocarcinomas and five squamous cell carcinomas: four stage IIIB and 26 stage IV. Sixteen patients who showed an objective tumour response (partial response) were classified as tumour responders and the remainder as non-responders according to World Health Organisation criteria (table 1).

\section{Immunohistochemistry}

We evaluated TP expression using the PD-ECGF monoclonal antibody (Oncogene, Boston, Massachusetts, USA). Immunohistochemical staining was carried out using the streptavidin-biotin-peroxidase technique. Paraffin wax embedded tissue sections ( $4 \mu \mathrm{m}$ thick) were dewaxed and then incubated in $0.5 \% \mathrm{H}_{2} \mathrm{O}_{2}$ in methanol for 30 minutes. After washing in Tris buffered saline, sections were incubated in normal human serum (1/10 dilution) for 20 minutes. Sections were incubated with the primary antibody ( $1 / 200$ dilution) for 90 minutes. After washing in Tris buffered saline for five minutes, sections were incubated with biotinylated goat antimouse and antirabbit immunoglobulins for 30 minutes (Dako, Carpenteria, California, USA). After incubation with strept ABComplex/horseradish peroxidase (Dako) for 30 minutes, the peroxidase reaction was developed using diaminobenzidine (Dako) as chromogen and sections were counterstained with haematoxylin. Omission of the primary antibody was used as a negative control. Mononuclear cells were used as a positive internal control.

TP staining was evaluated in tumour cells and stromal cells including fibroblasts, endothelial cells, macrophages, and lymphocytes without knowledge of the clinicopathological features. The percentage of TP positive cells-cells with strong cytoplasmic and/or nuclear reactivity—was recorded for each case. Tumour TP staining was compared with the surrounding stroma and scored as follows: 0, no staining; 1 , $<50 \%$ of tumour cells or showing weaker staining than that of stroma; $2, \geqslant 50 \%$ of tumour cells showing similar intensity to that of stroma; and $3, \geqslant 50 \%$ of tumour cells showing stronger staining than that of stroma. For statistical analysis, a score of 2 or 3 was considered high tumour cell TP expression. Similarly, the degree of stromal TP expression

Table 2 Association between tumour cell TP expression and clinicopathological features of the patients

\begin{tabular}{|c|c|c|c|c|c|}
\hline \multirow[b]{2}{*}{ Clinicopathological features } & \multicolumn{5}{|c|}{ Tumour cell TP expression $(n=30)$} \\
\hline & High $(n=13)$ & $\%$ & Low $(n=17)$ & $\%$ & p Value \\
\hline \multicolumn{6}{|l|}{ Histology } \\
\hline Adenocarcinoma $(n=25)$ & 11 & 44 & 14 & 56 & 1.0 \\
\hline Squamous cell carcinoma $(n=5)$ & 2 & 40 & 3 & 60 & \\
\hline \multicolumn{6}{|l|}{ Stage } \\
\hline$\| \mathrm{IIB}(\mathrm{n}=4)$ & 0 & 0 & 4 & 100 & 0.13 \\
\hline IV $(n=26)$ & 13 & 50 & 13 & 50 & \\
\hline \multicolumn{6}{|l|}{ Sex } \\
\hline Male $(n=15)$ & 5 & 33 & 10 & 67 & 0.46 \\
\hline Female $(n=15)$ & 8 & 53 & 7 & 47 & \\
\hline \multicolumn{6}{|l|}{ Tumour response } \\
\hline Responder $(n=16)$ & 11 & 69 & 5 & 31 & $0.004^{*}$ \\
\hline Non-responder $(n=14)$ & 2 & 14 & 12 & 86 & \\
\hline
\end{tabular}




\begin{tabular}{|c|c|c|c|c|c|}
\hline \multirow[b]{2}{*}{ Clinicopathological features } & \multicolumn{5}{|c|}{ Stromal TP expression $(n=28)$} \\
\hline & High $(n=16)$ & $\%$ & Low $(n=12)$ & $\%$ & $\mathrm{p}$ Value \\
\hline \multicolumn{6}{|l|}{ Histology } \\
\hline Adenocarcinoma $(n=23)$ & 13 & 57 & 10 & 43 & \multirow[t]{2}{*}{1.0} \\
\hline Squamous cell carcinoma $(n=5)$ & 3 & 60 & 2 & 40 & \\
\hline \multicolumn{6}{|l|}{ Stage } \\
\hline IIIB $(n=4)$ & 4 & 100 & 0 & 0 & \multirow[t]{2}{*}{0.13} \\
\hline IV ( $n=24)$ & 12 & 50 & 12 & 50 & \\
\hline \multicolumn{6}{|l|}{ Sex } \\
\hline Male $(n=15)$ & 9 & 60 & 6 & 40 & \multirow[t]{2}{*}{1.0} \\
\hline Female $(n=13)$ & 7 & 54 & 8 & 46 & \\
\hline \multicolumn{6}{|l|}{ Tumour response } \\
\hline Responder $(n=15)$ & 5 & 33 & 10 & 67 & \multirow[t]{2}{*}{$0.009^{*}$} \\
\hline Non-responder $(n=13)$ & 11 & 85 & 2 & 15 & \\
\hline
\end{tabular}

was graded as low (diffuse weak expression in $0-50 \%$ of stroma) and high (diffuse strong expression in $>50 \%$ of stroma).

\section{Statistical analysis}

The $\chi^{2}$ and Fisher's exact tests were used to compare the baseline characteristics between the subgroups. Survival curves were calculated by the Kaplan-Meier method ${ }^{14}$ and compared by the log rank test.

\section{RESULTS}

\section{TP expression in NSCLC}

TP was primarily expressed in tumour cells and tumour associated macrophages and lymphocytes. The usual pattern of TP staining was cytoplasmic, although occasionally both cytoplasmic and nuclear staining was seen (fig 1). In tumour cells, high TP expression was seen in 13 of 30 cases (table 2), whereas in the stroma, TP expression was detected in 28 cases and high expression was seen in 16 cases (table 3 ). TP expression in tumour cells and stroma was not associated with tumour histology, stage, or sex.

We also used TP staining to assess the degree of tumour infiltration by macrophages as low and high. High macrophage infiltration was defined as macrophage infiltration in more than $50 \%$ of the examined optical fields. Macrophage infiltration was related inversely to TP expression in tumour cells $(p=0.02)$. High stromal TP expression was strongly related to a high degree of macrophage infiltration $(p=0.002$; table 4$)$, suggesting that the infiltrating macrophages were the major component of stromal TP expression.

Table 4 Association between TP expression in tumour cells and stroma and macrophage infiltration

\begin{tabular}{|c|c|c|c|}
\hline & \multicolumn{2}{|c|}{$\begin{array}{l}\text { Macrophage infiltration } \\
(n=28)\end{array}$} & \multirow[b]{2}{*}{ p Value } \\
\hline & High $(n=15)$ & Low $(n=13)$ & \\
\hline \multicolumn{4}{|c|}{$\begin{array}{l}\text { Tumour cell TP expression } \\
(n=28)\end{array}$} \\
\hline High $(n=12)$ & 3 & 9 & 0.02 \\
\hline Low $(n=16)$ & 12 & 4 & \\
\hline \multicolumn{4}{|c|}{ Stromal TP expression $(n=28)$} \\
\hline High $(n=16)$ & 13 & 3 & $0.002^{*}$ \\
\hline $\operatorname{Low}(n=12)$ & 2 & 10 & \\
\hline
\end{tabular}
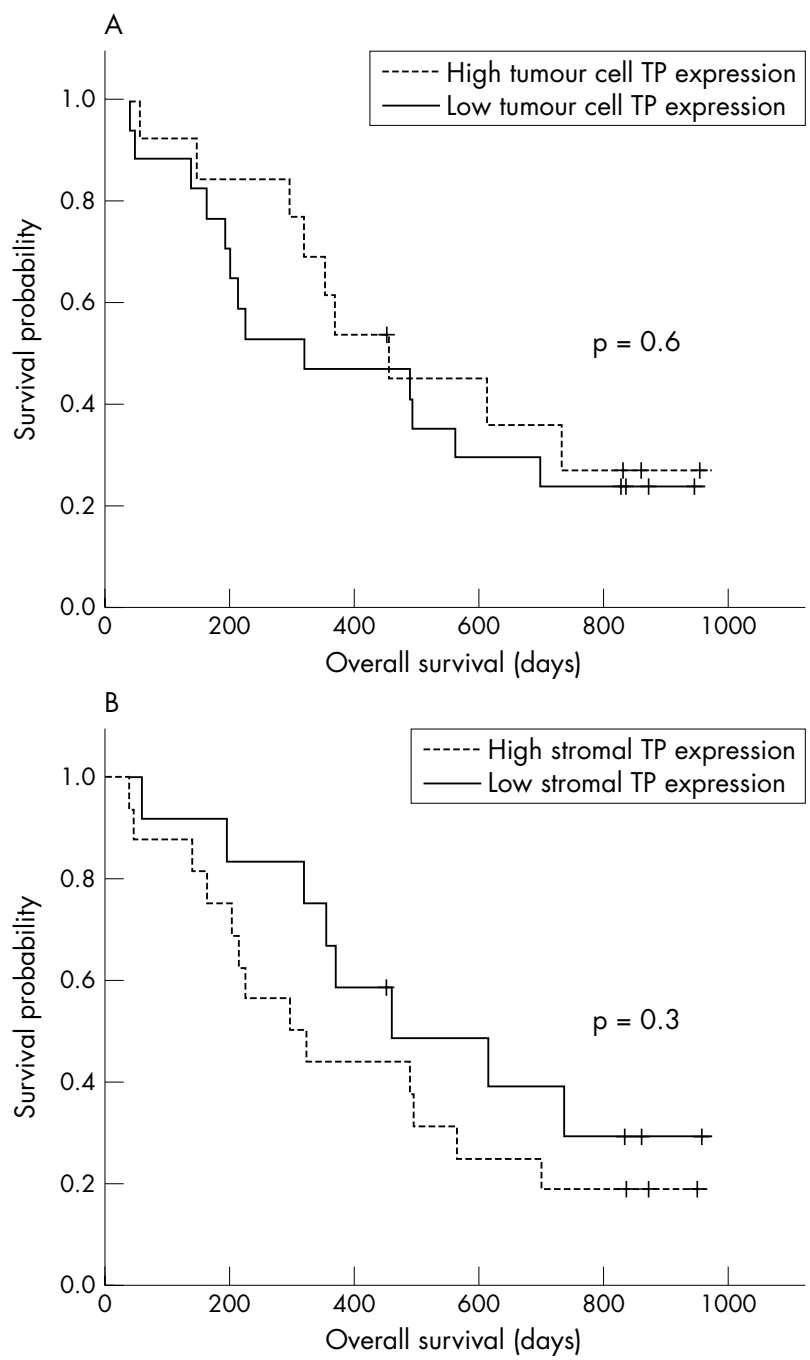

Figure 2 (A) Overall survival according to thymidine phosphorylase (TP) expression in tumour cells. (B) Overall survival according to TP expression in the stroma.

Relation between TP expression and tumour response and survival

We found a significant relation between tumour response to capecitabine plus docetaxel chemotherapy and TP expression. 
More responders had high tumour cell TP expression $(\mathrm{p}=0.004$; table 2$)$ and low stromal TP expression $(\mathrm{p}=0.009$; table 3$)$.

Because increased TP expression is associated with poor prognosis in various tumours, ${ }^{15}{ }^{16}$ we also investigated the prognostic value of TP expression. Although there was a tendency towards a better survival for patients with high tumour cell TP expression (fig 2A) and low stromal TP expression (fig $2 \mathrm{~B})$, the results were not significant $(\mathrm{p}=0.6$ and $\mathrm{p}=0.3$, respectively).

\section{Relation between TP expression and toxicity}

The most frequent toxicities in our clinical trial were moderate to severe stomatitis (33\%) and hand-foot syndrome (35\%). Haematological toxicities were uncommon; the prevalence of grade 3 or 4 neutropenia was $13 \%$. We also investigated the role of TP expression as a determinant of the toxicity of capecitabine based treatment and found that severe grade 3 or 4 hand-foot syndrome was more common in patients with high tumour cell TP expression $(\mathrm{p}=0.01$; fig $3 \mathrm{~A}$ ) and low stromal TP expression ( $\mathrm{p}=0.04$; fig $3 \mathrm{~B}$ ).

\section{DISCUSSION}

Because TP is involved in the final step of the conversion of capecitabine into 5-FU, tumours with high TP expression would be sensitive to capecitabine. ${ }^{12}$ However, the role of TP as a predictive marker for capecitabine based treatment in NSCLC is unknown. In this clinicopathological correlation

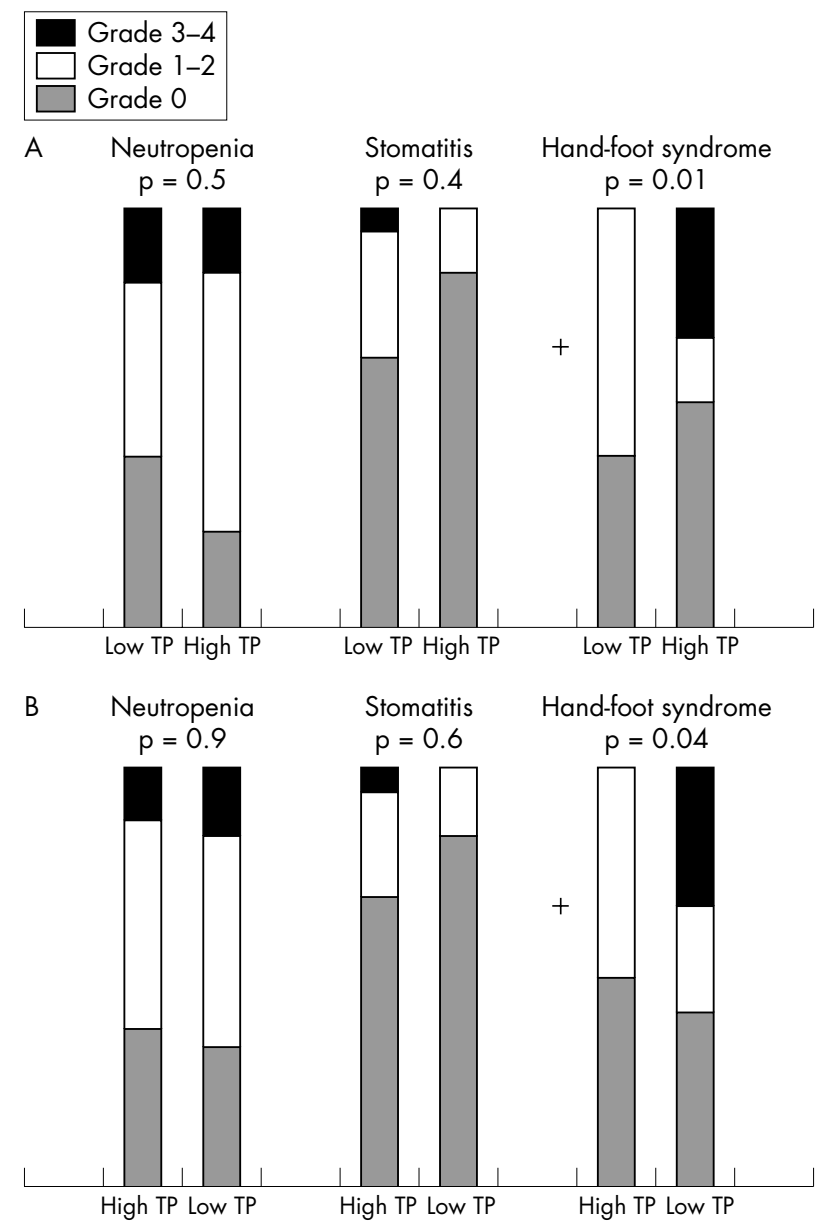

Figure 3 (A) Relation between thymidine phosphorylase (TP) expression in tumour cells and common toxicities. (B) Relation between TP expression in the stroma and common toxicities. analysis, we examined the predictive significance of TP expression in chemonaïve patients with advanced NSCLC who underwent capecitabine plus docetaxel chemotherapy. There was a significant association between tumour response to capecitabine plus docetaxel chemotherapy and TP expression in tumour cells.

Compared with adjacent normal tissues, increased TP expression is found in a variety of tumours including NSCLC. ${ }^{8}{ }^{17}$ Although the basic mechanism of this tumour specific increase in TP is not well understood, inflammatory cytokines such as interleukin 1, tumour necrosis factor, interferon $\alpha$, and interferon $\gamma$ are known to upregulate TP expression in various cell lines. ${ }^{18}$ Moreover, hypoxia and low $\mathrm{pH}$ also result in upregulation of TP expression. ${ }^{19}$ Several recent studies have reported that macrophages are the predominant cells stained positive for TP in tumour tissue, ${ }^{20-22}$ suggesting that macrophages activated by inflammatory cytokines and microenvironmental factors might be the source of TP expression in tumour tissue.

Furthermore, infiltration of TP positive macrophages is closely related to poor prognosis because it is associated with angiogenesis and an aggressive phenotype. ${ }^{20}$ Tumour associated macrophage infiltration is known to stimulate angiogenesis by the production of various factors including vascular endothelial growth factor, tumour necrosis factor $\alpha$, and TP. ${ }^{23}$ In breast cancer, TP positive macrophage infiltration is highly correlated with increased vascular endothelial growth factor expression and microvessel density. ${ }^{23}$ Therefore, the presence of TP positive macrophages might be characteristic of a more aggressive tumour environment and poor prognosis.

"In summary, our results support the hypothesis that nonsmall cell lung cancers with high tumour cell thymidine phosphorylase expression show a better response to capecitabine based chemotherapy"

In our current study, we found that the tumour response to capecitabine plus docetaxel chemotherapy was inversely related to stromal TP expression (mainly resulting from TP positive macrophages) $(p=0.009)$. Although the results were not significant, patients with high stromal TP expression tended to have a worse prognosis. In contrast, TP expression in tumour cells was closely associated with tumour response $(p=0.004)$. In addition, tumour cell TP expression was inversely related to TP positive macrophage infiltration $(\mathrm{p}=0.02)$ and patients with high tumour cell TP expression tended to have a better prognosis. These findings suggest that stromal TP expression associated with TP positive macrophage infiltration is characteristic of an

\section{Take home messages}

- In advanced non-small cell lung cancer (NSCLC), stromal thymidine phosphorylase (TP) expression associated with TP positive macrophage infiltration appeared to be characteristic of an aggressive phenotype with a worse prognosis

- In contrast, tumour cell TP expression, which is inversely related to the presence of TP positive macrophages, might have a role in predicting better tumour response to capecitabine based chemotherapy and prognosis in NSCLC

- A large scale prospective study is needed to confirm the prognostic significance of TP expression in NSCLC 
aggressive phenotype of NSCLC and could be a poor prognostic factor. However, TP expression in tumour cells, which is inversely related to the presence of TP positive macrophages, might have a role in predicting better tumour response to capecitabine based chemotherapy and prognosis in NSCLC.

The influence of tumour cell TP expression on fluoropyrimidine toxicity has not been well defined. It is generally accepted that capecitabine has a higher therapeutic index than other fluoropyrimidines because it is preferentially activated by TP in tumour cells. ${ }^{12}$ However, when it was given with docetaxel, which upregulates TP expression, considerable toxicity was seen in patients with metastatic breast cancer. ${ }^{7}$ We also saw enhanced toxicity during chemotherapy: hand-foot syndrome was closely related to TP expression in tumour cells, but was inversely related to stromal TP expression, suggesting that docetaxel mediated upregulation of TP might influence the concentration of TP in tumour cells and result in increased tumour response and toxicity.

In summary, our results support the hypothesis that NSCLCs with high tumour cell TP expression show a better response to capecitabine based chemotherapy. However, NSCLCs with high stromal TP expression and TP positive macrophages appear to have a poor prognosis, suggesting a more aggressive phenotype.

Because the patients in our current study had advanced NSCLC and were not undergoing surgery, we had difficulty in obtaining large tissue samples, which limited further evaluation of the relation between TP expression and other prognostic factors. Therefore, studies using surgical samples would be necessary to confirm the prognostic significance of TP expression in NSCLC. Furthermore, the relation between TP expression in tumour cells and/or stroma and angiogenesis needs to be clarified in the future.

\section{ACKNOWLEDGEMENTS}

This work was supported by National Cancer Centre Grant 0210140 from National Cancer Centre, Korea. Capecitabine was provided by Roche, Korea.

\section{Authors' affiliations}

J-Y Han, E K Hong, S Y Lee, S M Yoon, D H Lee, J S Lee, Research Institute and Hospital, National Cancer Centre, Goyang, Gyeonggi, 809 Madul-dong, Ilsan-gu, Goyang-si, Gyeonggi-do, 41 1-769, Korea

\section{REFERENCES}

1 Miwa M, Ura M, Nishida $M$, et al. Design of a novel oral fluoropyrimidine carbamate, capecitabine, which generates 5-fluorouracil selectively in tumours by enzymes concentrated in human liver and cancer tissue. Eur J Cancer 1998;34:1274-81.
2 Schuller J, Cassidy J, Dumont E, et al. Preferential activation of capecitabine in tumor following oral administration in colorectal cancer patients. Cancer Chemother Pharmacol 2000;34:291-7.

3 Morita T, Matsuzaki A, Tokue A. Enhancement of sensitivity to capecitabine in human renal carcinoma cells transfected with thymidine phosphorylase cDNA. Int J Cancer 2001;92:451-6.

4 Sawada N, Ishikawa T, Fukase $Y$, et al. Induction of thymidine phosphorylase activity and enhancement of capecitabine efficacy by taxol/taxotere in human cancer xenografts. Clin Cancer Res 1998:4:1013-19.

5 Fujimoto-Ouchi K, Tanaka Y, Tominaga T. Schedule dependency of antitumor activity in combination therapy with capecitabine/ $/ 5^{\prime}$-deoxy-5-fluorouridine and docetaxel in breast cancer models. Clin Cancer Res 2001;7:1079-86.

6 Kurosumi M, Tabei T, Suemasu K, et al. Enhancement of immunohistochemical reactivity for thymidine phosphorylase in breast carcinoma cells after administration of docetaxel as a neoadjuvant chemotherapy in advanced breast cancer patients. Oncol Rep 2000;7:945-8.

7 O'Shaughnessy J, Miles D, Vukelja S, et al. Superior survival with capecitabine plus docetaxel combination therapy in anthracycline-pretreated patients with advanced breast cancer: phase III trial results. J Clin Oncol 2002;20:2812-23.

8 Giatromanolaki A, Koukourakis MI, Comley M, et al. Platelet-derived endothelial cell growth factor (thymidine phosphorylase) expression in lung cancer. J Pathol 1997;181:196-9.

9 J-Y Han, DH Lee, HY Kim, et al. A phase II study of weekly docetaxel plus capecitabine for patients with advanced non-small cell lung cancer (NSCLC). Cancer 2003;98:1918-24.

10 Cerny T, Kaplan S, Pavlidis N, et al. Docetaxel (Taxotere) is active in non-small cell lung cancer: a phase II trial of EORTC early clinical trials group (ECTG). Br J Cancer 1994;70:384-7.

11 Francis PA, Rigas JR, Kris MG, et al. Phase II trial of docetaxel in patients with stage III and IV non-small cell lung cancer. J Clin Oncol 1997:12:1232-7.

12 Miller VA, Rigas JR, Francis PA, et al. Phase II trial of a $75 \mathrm{mg} / \mathrm{m}^{2}$ dose of docetaxel with prednisone premedication for patients with advanced nonsmall cell lung cancer. Cancer 1995;75:968-72.

13 Kunitoh H, Watanabe K, Onoshi T, et al. Phase II trial of docetaxel in previously untreated advanced non-small-cell lung cancer: a Japanese cooperative study. J Clin Oncol 1996;14:1649-55.

14 Kaplan ES, Meier P. Non-parametric estimation from incomplete observation. $J$ Am Stat Assoc 1958;53:457-81.

15 Takao S, Takebayashi Y, Che X, et al. Expression of thymidine phosphorylase is associated with a poor prognosis in patients with ductal adenocarcinoma of the pancreas. Clin Cancer Res 1998;4:1619-24.

16 Sato J, Sata $M$, Nakamura $H$, et al. Role of thymidine phosphorylase on invasiveness and metastasis in lung adenocarcinoma. Int $J$ Cancer 2003;106:863-70

17 Takebayashi Y, Yamada K, Miyadera K, et al. The activity and expression of thymidine phosphorylase in human solid tumours. Eur J Cancer 1996;32:1227-32.

18 Eda H, Fujimoto K, Watanabe S, et al. Cytokines induce thymidine phosphorylase expression in tumor cells and make them more susceptible to 5'-deoxy-5-fluorouridine. Cancer Chemother Pharmacol 1993;32:333-8.

19 Griffiths L, Dachs GU, Bicknell R, et al. The influence of oxygen tension and pH on the expression of platelet-derived endothelial cell growth factor/thymidine phosphorylase in human breast tumor cells grown in vitro and in vivo. Cancer Res 1997;57:570-2.

20 Yao Y, Kubota T, Sato K, et al. Macrophage infiltration-associated thymidine phosphorylase expression correlates with increased microvessel density and poor prognosis in astrocytic tumors. Clin Cancer Res 2001;7:4021-6.

21 Toi $M$, Ueno T, Matsumoto $H$, et al. Significance of thymidine phosphorylase as a marker of protumor monocytes in breast cancer. Clin Cancer Res 1999;5:1131-7.

22 Leek RD, Landers R, Fox SB, et al. Association of tumour necrosis factor $\alpha$ and its receptors with thymidine phosphorylase expression in invasive breast carcinoma. Br J Cancer 1998;77:2246-51.

23 Leek RD, Hunt NC, Landers RJ, et al. Macrophage infiltration is associated with VEGF and EGFR expression in breast cancer. J Pathol 2000;190:430-6. 